\title{
The role of diagnostic cardiac catheterization for children with congenital heart diseases: local experience
}

\author{
Reham Wagdy
}

Department of Pediatrics, Faculty of Medicine, Alexandria University, Alexandria, Egypt

Submitted: 7 April 2018

Accepted: 23 April 2018

Arch Med Sci Atheroscler Dis 2018; 3: e72-e79

DOI: https://doi.org/10.5114/amsad.2018.76824

Copyright @ 2018 Termedia \& Banach

\section{Abstract}

Introduction: Despite the development of non-invasive tools of investigations for congenital heart diseases (CHDs), still the role of diagnostic cardiac catheterization (DCC) cannot be undermined. The study aimed to analyze the clinical profile of indicated CHDs cases at our center for DCC to evaluate the contribution of DCC in patients' management plans.

Material and methods: The study checked files of cases performed DCC between 2011 and 2012 at the pediatric catheterization unit of Alexandria University Children's Hospital by the same operator. Demographic, laboratory and hemodynamic data were collected and analyzed.

Results: Files of 61 children were included in the study. They were grouped into: group I (GI) $(n=25)$ with pulmonary hypertension $(\mathrm{PH})$, and group II (GII) $(n=36)$ cases with obstructive pulmonary artery diseases. For the PH group, ventricular septal defect (VSD) was the most common (55\%), and patients' outcome plans were based on angiography - operable subgroup GI-A: $(84 \%)(n=21)$ and non-operable $(16 \%)$ GI-B: (4\% inoperable, $12 \%$ recommended for sildenafil). GI-B cases were significantly older, with higher PVRI and PVRI/SVRI $(3.62,0.68, p=0.002)$ compared to GI-A $(0.89,0.23$, $p=0.002$, respectively). For group II, tetralogy of Fallot (TOF) was predominant $(60 \%)$ among the disease spectrum, and the McGoon ratio ranged from 0.7 to 3.2 (median: 1.8). Outcome for Gll: $63 \%$ operable, $25 \%$ shunt, $12 \%$ stent. Only pulmonary atresia cases showed a significant difference in the unmatched plans between echocardiography and catheterization reports $\left(\chi^{2}=7.438, p=0.023\right)$.

Conclusions: Diagnostic cardiac catheterization had a positive contribution for determination of the management plans for more than $84 \%$ of cases. The utility of pediatric cardiac catheterization in diagnosis should be adjusted according to the local needs of every cardiac center.

Key words: cardiac angiography, congenital heart diseases, pulmonary vascular resistance index, hemodynamic data.

\section{Introduction}

Despite the advances of noninvasive imaging modalities, the utility of pediatric cardiac catheterization in diagnostic purposes is still the gold standard investigation for many congenital heart diseases (CHDs). Echocardiography is the main non-invasive method for diagnosis of CHDs; however, it is not always sufficient for the cardiologist to assess operability or to predict the surgical outcome [1]. Cardiac magnetic resonance imaging (MRI) and computed tomography (CT) are powerful tools used to provide anatomical and physiological information; how-

\author{
Corresponding author: \\ Reham Wagdy \\ Department of Pediatrics \\ Faculty of Medicine \\ Alexandria University \\ 0217 Alexandria, Egypt \\ Phone: +966568654977 \\ E-mail: dr_reham_wagdy@ \\ yahoo.com
}


ever, their availability is not uniform at all centers [2, 3]. Many indications can be encountered for diagnostic cardiac catheterization (DCC). It is recommended to assess pulmonary vascular resistance (PVR) and reversibility of pulmonary hypertension (level of evidence: B) [4]. Recently, estimation of the pulmonary vascular resistance index (PVRI) instead has become the cornerstone for decision making for patient management according to the recent American Heart Association Guidelines (AHA) [5]. A baseline PVRI of $<6 \mathrm{WU} \cdot \mathrm{m}^{2}$ and a PVRI/ SVRI ratio $<0.3$ have been used as an indicator of operability systemic vascular resistance index (SVRI). Also, DCC is indicated for children with complex pulmonary atresia when the data about pulmonary artery anatomy, major aorto-pulmonary collateral arteries (MAPCAs) and coronary circulation from other methods are equivocal $[4,6]$. Meanwhile, with level of evidence C, postoperative DCC is reasonable when the early post-operative course is unexpectedly complicated and MRI and CT angiography fail to yield a clear explanation. Although diagnostic cardiac catheterization can offer valuable data about structure and hemodynamics of the underlying cardiac lesions, it should not be considered routine for diagnosis of congenital defects. Individual centers must assess their abilities for determining which catheterization cases they might perform [4].

We were motivated to conduct this study to assess the main local indications of diagnostic catheterization in our center and the patients' clinical profile with the role of DCC in determining their managements plans.

\section{Material and methods}

\section{Ethical considerations}

Ethical approval was obtained from the Ethical Review Committee, Alexandria University on 18/ May/2017 with serial number 0303637.

\section{Study design, settings and population}

The current study was a retrospective descriptive cross sectional study. It checked all the medical records of children catheterized by the same operator for diagnostic purposes between January 2011 and July 2012 at the cardiac catheterization laboratory of the pediatric department at Alexandria University Children's Hospital, which is considered a referral center for many hospitals. Catheter-based intervention cases were excluded.

\section{Sample size}

As a descriptive study it concentrated on continuous variables, i.e. variable diseases and variable outcomes. Hence, the sample size of the proposed study was 61 cases [7].

\section{Methods}

Detailed history, clinical examinations, laboratory results and oxygen saturation data were collected. Basically, our center was lacking cardiac MRI and CT. Therefore, echocardiography was the only prior imaging modality before catheterization. Conventional echocardiography reports were analyzed for the diagnosis, cardiac function, chamber dilatations, coronaries anomalies and pressure gradients specially across tricuspid and pulmonary valves whether for regurgitations or stenosis. The machine was a Madison 990 (2 and $5 \mathrm{MHz}$ transducers).

\section{Cardiac catheterization}

It was performed at the pediatric cardiac catheterization unit. Before the procedure, the patients and their relatives were emotionally prepared and informed consent forms were signed. Children were kept fasting for 4-5 h except for regularly scheduled oral medications. At the time of the procedure, general anesthesia was induced by intravenous administration of propofol and ketamine, then it was maintained by inhalation of halothane. Venous and arterial access were obtained after puncturing femoral vessels by the Seldinger technique. After advancement of sheath and dilators (no. 5-6 Fr) heparin (100 U/kg) was given at the beginning of the procedure. NIH catheters were the usual used catheters for right heart catheterization while a pigtail catheter was used for the left side of the heart. A complete profile of the hemodynamic state of the heart was assessed including pressures and obtaining samples for blood gases. Different cineangiographic pictures were recorded in anteroposterior and lateral views (biplane projection) after injection of non-ionic contrast material (Omnipaque) from a flow injector $(1 \mathrm{ml} / \mathrm{kg})$ at a rate of $1 \mathrm{ml} / \mathrm{s}$. After the procedure children were given antibiotics as a routine prophylactic measure and monitored for the next $12 \mathrm{~h}$ then discharged [8].

The reports were checked to confirm the diagnosis and evaluate hemodynamic data as mean pulmonary artery pressure (PAP), PVR, systemic vascular resistance (SVR), PVRI, PVRI/SVRI and Qp : Qs (ratio of arterial pulmonary flow to systemic blood flow). The reference values of these parameters were checked according to AHA guidelines $[4,5]$. Also, cineangiographic pictures were analyzed to assess chamber size, obstructive lesions, shunts, MAPCAs and coronaries anomalies and to calculate McGoon's ratio for tetralogy of Fallot cases. It was calculated by dividing the sum of the diameters of right pulmonary artery (RPA) and left pulmonary artery (LPA) by the diameter of the aorta at the level above the diaphragm (RPA 
Table I. Clinical profile of CHDs of the studied groups

\begin{tabular}{|lccc|}
\hline Parameter & Number & $\begin{array}{c}\text { \% in } \\
\text { same } \\
\text { group }\end{array}$ & $\begin{array}{c}\text { \% in total } \\
\text { group } \\
(n=61)\end{array}$ \\
\hline \begin{tabular}{l} 
Group I $(n=25):$ \\
\hline VSD
\end{tabular} & 15 & 60 & 24.5 \\
\hline CAVCDs & 5 & 20 & 8.1 \\
\hline DORV & 3 & 12 & 4.9 \\
\hline TGA/ASD/VSD & 2 & 8 & 3.2 \\
\hline Group II $(n=31):$ & 17 & 47.2 & 27.2 \\
\hline TOF & 3 & 8.3 & 4.9 \\
\hline TOF/PA & 9 & 25 & 14.7 \\
\hline VSD/PS & 5 & 13.9 & 8.1 \\
\hline PS/LPA & 1 & 2.8 & 1.6 \\
\hline TGA/PS & 1 & 2.8 & 1.6 \\
\hline DCRV & 1 & & \\
\hline
\end{tabular}

VSD - ventricular septal defect, CAVCDs - complete atrioventricula septal defects, TOF - tetralogy of Fallot, PA - pulmonary atresia, ASD - atrial septal defect, PS - pulmonary stenosis, TGA transposition of great arteries, DORV - double outlet right ventricle, $D C R V$ - double chamber right ventricle.

$+\mathrm{LPA} / \mathrm{AO}))$. An average value of 2.1 was noted in normal subjects. A ratio above 1.2 is associated with acceptable postoperative RV systolic pressure in tetralogy of Fallot. A ratio below 0.8 is deemed inadequate for complete repair of pulmonary atresia cases with TOF anatomy (TOF/PA) $[6,9]$.

\section{Statistical analysis}

Data were fed to the computer and analyzed using IBM SPSS software package version 20.0. Comparisons between groups for categorical variables were assessed using the $\chi^{2}$ test. The Mann-Whitney test was used to compare two groups for abnormally distributed quantitative variables. Student's t-test was used for normally distributed quantitative variables, to compare between two studied groups. Significance of the obtained results was judged at the $5 \%$ level. Qualitative data were described using number and percent, while normally quantitative data were expressed as mean \pm SD, and abnormally distributed data were expressed as median (min.-max.).

\section{Results}

The medical records of 61 children subjected to DCC were checked and included in the study. Patients' ages ranged from 4 month to 144 months (median: 26 months). The enrolled cases were categorized into 2 main groups: group I $(n=25)$ included cases suffering from pulmonary hypertension with intracardiac shunts based on clinical and echocardiographic diagnosis; and group II $(n=36)$ included children suffering from different forms of obstructive pulmonary diseases with or without cyanosis, as summarized in Table I. Ventricular septal defects (VSD) were the most common diagnosis (60\%) among Gl, especially the perimembranous type, while tetralogy of Fallot (TOF) was the most frequent diagnosis among GII (55.5\%). However, TOF cases were predominant among the studied patients (27.2\%) (Table I).

The majority of patients included in Gl were be-tween 1 and 2 years of age and of VSD type while below 1 year the cases varied between VSD and TGA/VSD (transposition of great arteries). Cases above 4 years (12\%) were mostly suffering from TOF or complete atrioventricular septal defects (CAVCDs). Mostly oxygen saturation $\left(\mathrm{O}_{2}\right.$ sat) for $\mathrm{Gl}$ patients ranged from $78 \%$ to $98 \%$,

Table II. Distribution of the studied cases according to demographic data

\begin{tabular}{|c|c|c|c|}
\hline Data & Group I $(n=25)$ & Group I $(n=36)$ & Total number $(n=61)$ \\
\hline Age, median (range) [months]: & $24(4-120)$ & $30(6-144)$ & $26(4-144)$ \\
\hline No. of cases $\leq 12 \mathrm{~m}$ & $6(24 \%)$ & $7(19.4 \%)$ & $13(21.3 \%)$ \\
\hline No. of cases > 12-24 & $9(36 \%)$ & 7 (19.4\%) & $16(26.2 \%)$ \\
\hline No. of cases > 24-36 & $5(20 \%)$ & $6(16.7 \%)$ & $11(18 \%)$ \\
\hline No. of cases > 36-48 & $2(8 \%)$ & $4(11.1 \%)$ & $6(9.8 \%)$ \\
\hline No. of cases $>48$ & $3(12 \%)$ & $12(33.3 \%)$ & $15(24.6 \%)$ \\
\hline Weight, median (range) [kg] & $12(4-30)$ & $11(6-36)$ & $11(4-36)$ \\
\hline Height, mean (range) $[\mathrm{cm}]$ & $80.8 \pm 15.2$ & $89.4 \pm 22.9$ & $85.9 \pm 20.4$ \\
\hline BMI, median (range) $\left[\mathrm{kg} / \mathrm{m}^{2}\right]$ & $17.4(10.4-35.8)$ & $15.9(12.8-32)$ & $16.1(10.4-35.3)$ \\
\hline Median $\mathrm{SAO}_{2} \%$ (room air) & $94(78-98)$ & $90.5(70-100)$ & $93(70-100)$ \\
\hline
\end{tabular}


as shown in Table II. It was below $85 \%$ in $12 \%$ ( $n=3$ cases). Three patients were confirmed to be Down syndrome (trisomy 21). Echocardiography estimated the pressure gradient of tricuspid and pulmonary regurgitation (PGTR and PGPR) for 17 cases among group I with mean $40 \pm 10.8$ and $29.7 \pm 7.5$ respectively, as summarized in Table III. Cardiac catheterization hemodynamic data of $\mathrm{Gl}$ after giving $100 \%$ oxygen (vaso-reactivity test VRT) were summarized in Table III. The mean of PAP was $46.4 \pm 15.4$. However, 2 cases had a mean PAP of less than $25 \mathrm{~mm} \mathrm{Hg}$. The pulmonary to systemic blood flow ratio (Qp : Qs) had a mean of $1.74 \pm 0.80$ for all Gl patients; Qp : Qs was $>3$ for 2 patients, Qp : Qs ranged from 2 to 3 for 10 cases, $>1$ for 7 cases and Qp : Qs $=1$ for 2 cases. The PVRI had a wide range from 0.42 to 9.17 with a median of 0.93 . Also, the PVRI/SVRI ranged from 0.11 to 0.91 with a median of 0.25 . About $84 \%$ of cases $(n=21)$ had PVRI $<6 \mathrm{WU} \cdot \mathrm{m}^{2}$ and PVRI/ SVRI $<0.3$, while $1(4 \%)$ case remained with PVRI; 9.16 WU $\mathrm{m}^{2}$ and PVRI/SVRI; 0.91. Three (12\%) cases had PVRI/SVRI > 0.3; $(0.86,0.48,0.39$ respectively) but the PVRI was $<6 \mathrm{WU} \cdot \mathrm{m}^{2}$.

After analysis of catheterization reports and according to the AHA guidelines the patients' management plans ranged between operable conditions, inoperable cases and cases recommended for sildenafil therapy as summarized in Table IV. Comparing the demographic, echocardiographic and catheterization data of the operable and non-operable subgroups revealed a statistically significant difference between the two subgroups in age as the inoperable group included older children (median: 57 vs. 24, $p=0.014$ ). Echocardiography showed a significant difference for higher PGPR for non-operable when compared to operable patients ( $36.3 \pm 8.5$ vs. $28.2 \pm 6.6, p=0.049$ )

Table III. Comparison between operable (GI-A) and non-operable (GI-B) PH subgroups according to different parameters

\begin{tabular}{|c|c|c|c|c|c|}
\hline Parameter & Total $(n=25)$ & Group I-A $(n=21)$ & Group I-B $(n=4)$ & Test of sig. & $P$-value \\
\hline \multicolumn{6}{|l|}{ Gender: } \\
\hline Male & $11(44 \%)$ & $9(42.9 \%)$ & $2(50 \%)$ & $\chi^{2}=0.070$ & 1.00 \\
\hline Female & $14(56 \%)$ & $12(57.1 \%)$ & $2(50 \%)$ & & \\
\hline Age [months] & $24(4-120)$ & $24(4-60)$ & $57(30-120)$ & $U=9.00^{*}$ & $0.014^{\star}$ \\
\hline Weight [kg] & $12.2 \pm 5.1$ & $11.1 \pm 3.1$ & $18.4 \pm 9$ & $t=1.600$ & 0.204 \\
\hline Height $[\mathrm{cm}]$ & $81.2 \pm 15.4$ & $77.2 \pm 11.4$ & $102 \pm 18.2$ & $t=3.629^{*}$ & $0.001^{*}$ \\
\hline O sat $\%$ & $90.5 \pm 5.7$ & $91.3 \pm 5.5$ & $86.5 \pm 5.4$ & $t=1.582$ & 0.128 \\
\hline \multicolumn{6}{|l|}{ Echocardiography report: } \\
\hline PGTR [mm Hg] & $40.5 \pm 10.8$ & $38.6 \pm 10$ & $49 \pm 11.9$ & $t=1.840$ & 0.081 \\
\hline PGPR [mm Hg] & $29.7 \pm 7.5$ & $28.2 \pm 6.6$ & $36.3 \pm 8.5$ & $t=2.101^{*}$ & $0.049^{*}$ \\
\hline IVG [mm Hg] & $26.6 \pm 8.8$ & $26.2 \pm 8.4$ & $28.5 \pm 11.8$ & $t=0.473$ & 0.641 \\
\hline \multicolumn{6}{|l|}{ Catheterization data: } \\
\hline $\mathrm{RV}$ pressure $[\mathrm{mm} \mathrm{Hg}]$ & $69.1 \pm 15.3$ & $65.5 \pm 12.7$ & $88 \pm 14.9$ & $t=3.170^{*}$ & $0.004^{*}$ \\
\hline Sys/PAP [mm Hg] & $65.8 \pm 18.2$ & $59.8 \pm 12.3$ & $97.5 \pm 6.9$ & $t=5.890^{*}$ & $<0.001^{*}$ \\
\hline Mean PAP [mm Hg] & $46.4 \pm 15.4$ & $42.2 \pm 12.7$ & $68.3 \pm 8.4$ & $t=3.912^{*}$ & $0.001^{*}$ \\
\hline $\mathrm{Qp}: \mathrm{Qs}$ & $1.74 \pm 0.80$ & $1.92 \pm 0.75$ & $0.81 \pm 0.11$ & $t=6.429^{*}$ & $<0.001^{*}$ \\
\hline PVRI/SVRI & $0.25(0.11-0.91)$ & $0.23(0.11-0.30)$ & $0.68(0.40-0.91)$ & $U=0.0^{*}$ & $0.002^{*}$ \\
\hline PVRI [WU· $\left.\mathrm{m}^{2}\right]$ & $0.93(0.42-9.17)$ & $0.89(0.42-1.62)$ & $3.62(3.08-9.17)$ & $U=0.0^{*}$ & $0.002^{*}$ \\
\hline SVR [WU] & $9.9(4.5-14.3)$ & $9.2(5.2-12.8)$ & $11.4(4.5-14.3)$ & $U=31.00$ & 0.415 \\
\hline SVRI $\left[\mathrm{WU} \cdot \mathrm{m}^{2}\right]$ & $4.8 \pm 1.9$ & $4.3 \pm 1.4$ & $7.3 \pm 2.4$ & $t=3.643^{*}$ & $0.001^{*}$ \\
\hline PVR [WU] & $1.9(1.2-11.8)$ & $1.8(1.2-3.4)$ & $6(3.9-11.8)$ & $U=0.0^{*}$ & $0.002^{*}$ \\
\hline
\end{tabular}

$Q p$ - pulmonary vascular resistance, $Q s$ - systemic vascular resistance, PAP - pulmonary artery pressure, PVRI - pulmonary vascular resistance index, SVRI - systemic vascular resistance index. PGTR - pressure gradient of tricuspid regurgitation, PGPR - pressure gradient of pulmonary regurgitation, IVG - interventricular pressure gradient. $\chi^{2}, p-\chi^{2}$ and $p$-values for chi-square test for comparing between the two groups. $t, p$-t and $p$-values for Student's $t$-test for comparing between the two groups. $U, p-U$ and $p$-values for Mann-Whitney test for comparing between the two groups. *Statistically significant at $p \leq 0.05$. 
Table IV. Patient outcome after analysis of catheterization report

\begin{tabular}{|c|c|c|}
\hline Patient outcome & $N(\%)$ & Analysis of catheterization report \\
\hline \multicolumn{3}{|l|}{ Group I: } \\
\hline Operable & $21(84)$ & VSD, CAVCD, TGA, DORV \\
\hline Sildenafil therapy 6 months & $3(12)$ & 2 VSD/1 CAVCD \\
\hline Inoperable & $1(4)$ & PM wide VSD \\
\hline \multicolumn{3}{|l|}{ Group II: } \\
\hline Operable & $23(63.8)$ & 9 TOF, 9 VSD/PS, 3 ASD/PS, 1 DCRV \\
\hline Palliative shunt & $9(25)$ & 3 TOF/PA, 6 TOF MG $<1.2$ \\
\hline Stent dilatation & $4(11.1)$ & 2 PS/LPAS, 2 TOF + bifurcation stenosis \\
\hline
\end{tabular}

as summarized in Table III. The mean of PVR, PVRI, PVRI/SVRI, RV and PAP pressures were higher for non-operable cases (GI-B) when compared to operable subgroup GI-A with significant difference. Meanwhile, Qp : Qs results were significantly lower for non-operable cases $(<1)$ when compared to the operable subgroup, as summarized in Table III.

On the other hand, Gll included patients with obstructive pulmonary artery defects. About 33.3\% of the cases were above 4 years and the diagnosis varied between classic TOF, PS/ASD, PS/VSD and DCRV. Meanwhile $19.2 \%$ were below one year with different diagnosis as TGA/PS, TOF/PA and PS with left pulmonary branch ostial stenosis and with O sat below $87 \%$ (Tables I and II). After checking cineangiographic pictures, the McGoon ratio was calculated for all TOF cases as a predictor of operability. It ranged from 0.7 to 3.2 (median: 1.8 ); 8 cases had a ratio below 1.8. Table IV summarized the patients' outcome of Gll for different plans (operable, palliative shunt or stenting before operation).

Comparing the management plans for Gll cases based only on echocardiographic findings to the plans based on cardiac catheterization resulted in some matched and unmatched cases for every diagnosis, as summarized in Table V. There was a statistically significant difference between matched/unmatched plans based on echocardi- ography and catheterization concerned for TOF/PA cases $\left(\chi^{2}=7.438, p=0.023\right)$. Whereas, other CHDs included in Gll there were no significant difference between the matched and unmatched reports of echocardiography and catheterizations.

\section{Discussion}

Advances in cardiac care worldwide have allowed the repair of CHDs to take place from infancy or even during the neonatal period for many patients, before the development of complications. However, in developing countries the surgical management of a lot of cases becomes delayed to childhood as they have no access to cardiovascular care [10]. Although the utility of diagnostic cardiac catheterization in the clinical setting has diminished over the last years, is still constitutes a valuable tool in some aspects in the workup of CHDs, especially for older children $[2,3]$. The current study focused on studying the local indications of DCC at our center in order to reflect the impact of that on our patients' management. It is important to point out that our center lacked CT and MRI; echocardiography was the only available non-invasive imaging method.

In our study, medical files of 61 patients were revised and grouped into a $\mathrm{PH}$ group and obstructive pulmonary artery disease group. The $\mathrm{PH}$ group

Table V. Distribution of matched and unmatched echo reports to catheterization reports for different cases among group II

\begin{tabular}{|lccccc|}
\hline Diagnosis & Total $(n=36)$ & Unmatched $(n=11)$ & Matched $(n=25)$ & $\chi^{2}$ & $P$-value \\
\hline TOF & $17(47.2 \%)$ & $5(45.5 \%)$ & $12(48.0 \%)$ & 0.020 & 0.888 \\
\hline TOF/PA & $3(8.3 \%)$ & $3(27.3 \%)$ & $0(0.0 \%)$ & $7.438^{*}$ & $0.023^{*}$ \\
\hline TGA-PS & $1(2.8 \%)$ & $0(0.0 \%)$ & $1(4.0 \%)$ & 0.453 & 1.000 \\
\hline PS/LPA & $5(13.9 \%)$ & $2(18.2 \%)$ & $3(12.0 \%)$ & 0.244 & 0.631 \\
\hline DCRV & $1(2.8 \%)$ & $0(0.0 \%)$ & $1(4.0 \%)$ & 0.453 & 1.000 \\
\hline VSD + PS & $9(25.0 \%)$ & $1(9.1 \%)$ & $8(32.0 \%)$ & 2.138 & 0.223 \\
\hline
\end{tabular}

$\chi^{2}, p-\chi^{2}$ and $p$-values for chi-square test for comparing between the two groups. *Statistically significant at $p \leq 0.05$. 
included a spectrum of diseases. VSD cases were the predominant defect among the shunt group, similar to the conclusion of Saxena et al. [11], and also matched with an Egyptian study performed at Alexandria which stated the same, and it considered other shunts, ASD, PDA, CAVC and TGA, less common [12]. Meanwhile, TOF was the most common indication among the obstructive pulmonary bed group and was the most common diagnosis among the whole study group $(27.2 \%$ TOF vs. $24.5 \%$, VSD). However, that was inconsistent with Kumar, who found the ratio of TOF to VSD to be $1: 3$, and this may be attributed to delayed surgical correction of TOF cases in our country when compared to VSD cases [13]. This may be explained by the complex anatomy of TOF, requiring more skilled surgeons and better postoperative care. It was clearly evident through comparing the main age group of both cases at the time of DCC (> 4 years for $\mathrm{Gll}$ versus $1-2$ years for $\mathrm{Gl}$ ).

In our study, $\mathrm{PH}$ was documented by DCC for all cases except for 2 of them (6.6\%). The reports showed mean PAP $<25 \mathrm{~mm} \mathrm{Hg}$ after the vasoreactivity test and normal PVRI values which declared that they were not good candidates for DCC from the start. This was consistent with Groh et al., who reported that echocardiography inaccurately estimates right ventricular pressure in children, either overestimating or underestimating it [14]. Another meta-analysis study showed that pooled sensitivity and specificity of Doppler echocardiography for the diagnosis of $\mathrm{PH}$ were $88 \%$ and $56 \%$ [15]. However, this was contradictory to Sohrabi et al., who found a significant correlation between systolic PAP by catheterization and TR velocity by echocardiography with sensitivity $92.8 \%$ and specificity of $86.6 \%$ [16]. Therefore, although echo inaccurately estimates $\mathrm{PH}$, it still has acceptable specificity and sensitivity in detecting severe $\mathrm{PH}$. Those 2 patients had mild $\mathrm{PH}$ and also their ages were 6 and 8 months, with large VSD, which raised the value of the suggestion from the Taleb study; rare and remote possibility of elevated PVR below 1 year of age. This will be considered in our further indications for DCC to overcome unnecessary use [15].

After checking the hemodynamic data of the $\mathrm{PH}$ group, the plans of management were classified according to recent AHA guidelines into operable (84\%) and non-operable (16\%) (inoperable (4\%) and borderline inoperable (sildenafil therapy) (12\%) for 6 months then reassessment) [4]. Comparing the clinical profile of operable and non-operable subgroups revealed that the difference in the age of patients was significant among both, as operable children were younger, around 24 months, while non-operable children were older, around 57 months. Therefore, older patients $>2$ years may have higher risk for inoperability than younger ones, which mandates the indication of DCC prior to surgical corrections for better prediction of surgical outcome. Moreover, pressure gradient values of pulmonary regurgitation were significantly higher among the non-operable group, while pressure gradient values of tricuspid regurgitation showed no significant difference between the two groups, in disagreement with Sohrabi et al. maybe because of the small sample size [17].

Concerning the catheterization hemodynamic parameters, PVRI and resistance ratio were significantly higher for the non-operable group compared to the operable group [17]. One case was inoperable (Eisenmenger syndrome); Down syn-drome, 6 years and half, wide outlet type VSD, no history of prior pulmonary artery banding, PVRI $=9.2$ and resistance ratio $=0.7$. Whereas the other 3 cases with borderline/inoperability were recommended for sildenafil treatment for 6 months then reassessment [18]. The other patients were recommended for elective corrective surgery with a predicted favorable outcome.

Concerning group II, the obstructive PA group, TOF represented more than half the cases. The surgical repair strategy was dependent on ensuring adequate PA size and distal pulmonary vascular bed with exclusion of coronary anomalies and MAPCAs, for which there is considered limited ability by echocardiography, especially in older children and obese patients. Furthermore, echocardiography ability is better at assessing the right pulmonary artery branch than the left. This may be because the left pulmonary artery's posterior course is poorly visualized via transthoracic scanning view [10].

The $\mathrm{McGoon}$ ratio is a reliable parameter in predicting the operability of TOF as it is proportionate to pulmonary flow and considered a goal for DCC. Some studies admitted the useful role of $M R I$ in calculating the McGoon ratio non-invasively [19]. However, it takes a long time to perform MRI, needs fully cooperative or sedated subjects, produces a poorer quality image in cases of severe cardiac arrhythmia, is expensive and requires expertise in image acquisition $[2,3,19,20]$. Computed tomography has become capable of delineating the anatomy in multiple planes within $2 \mathrm{~min}$, with less radiation, especially in older patients, although some studies stated false positive data about MAPCAs [3, 21, 22]. As long as these modalities were not available, we were obliged to perform DCC for all TOF cases, even classic and those younger than 1 year.

After revising the DCC reports, the patients' management plans and operability were determined for Gll according to each CHD's pre-requisites for intervention. About $60 \%$ of cases of TOF were classic and had a McGoon ratio > 1.8 and 
were recommended for surgical correction as recommended by many studies for a favorable surgical outcome. However, the other $40 \%$ of TOF had either hypoplastic pulmonary vascular beds or significant MAPCS collaterals and the diagnosis varied between TOF/PA and classic TOF in older children ( $>4$ years), which affected their management plans between shunt or intervention for the collateral [6, 8, 22].

Our results matched the Kumar study in India, which found MAPCS in older children with classic TOF and recommended DCC in absence of CT or MRI before corrective surgery [23].

Stent dilation were recommended for PS/LPA stenosis. All cases of VSD/PS were operable as they had normal hemodynamics as pulmonary stenosis decreased the overwork over the right ventricle similar to pulmonary artery banding.

It was interesting to compare the matching or non-matching between the echocardiography and cardiac angiography reports for operable and inoperable subgroups II. It highlighted the superior role of DCC over echocardiography in certain conditions and the unnecessary indications of DCC for others. Concerning cases of TOF/PA, they had significantly unmatched echocardiography reports when compared to DCC, which mandates the DCC. This agreed with recommended guidelines and many studies [4, 10, 23]. Meanwhile, for classic TOF, VSD/PS, PS/LPA, TGA/PS and DCRV reports did not show a significant difference in matched and unmatched criteria to catheterization reports between operable and non-operable groups. Results of TGS/PS and DCRV cases were based on small samples (1 case each) so it deserves further studies on larger samples. Apart from classic TOF cases especially older children and PS cases specially with LPAs, all cases of VSD/PS were unnecessarily catheterized as long as the echocardiographic report showed left to right shunt at rest and exercise [24].

For our local center diagnostic cardiac cauterization had a positive contribution in decision making in medical or surgical management for about $85.2 \%$ of the indicated cases; however, about $14.8 \%$ of cases had no added benefits. This agreed with a similar Indian study conducted to assess the role of DCC in the patient's fate and had almost the same logistics of developing countries; they concluded the great importance for DCC in further management of over than $90 \%$ of their pediatric cases [23]. Also, it agreed with other studies in Pakistan and Sudan [10, 25].

In conclusion, the diagnostic role of pediatric cardiac catheterization remains the "final authority" for definitive anatomic and hemodynamic information. However, the utility and the indications should be adjusted according to the local circumstances of every center in order to achieve the maximum positive contribution for patients' management plans.

\section{Acknowledgments}

I would like to thank the nursing team of the pediatric catheterization laboratory at El-Shatby Hospital at Alexandria.

\section{Conflict of interest}

The author declares no conflict of interest.

\section{References}

1. Habib G, Torbicki A. The role of echocardiography in the diagnosis and management of patients with pulmonary hypertension. Eur Respir Rev 2010; 19: 288-99.

2. Ntsinjana HN, Hughes ML, Taylor AM. The role of cardiovascular magnetic resonance in pediatric congenital heart disease. J Cardiovasc Magn Reson 2011; 13: 51.

3. Chen BB, Chen, SJ, Hwan Wu MH, Li YW, L HC. EBCT McGoon ratio a reliable and useful method to predict pulmonary blood flow non-invasively. Chin J Radiol 2007; 32: 1-8.

4. Feltes TF, Bacha E, Beekman RH, et al. Indications for cardiac catheterization and intervention in pediatric cardiac disease. Circulation 2011; 23: 2607-52.

5. Abman SH, Hansmann G, Archer SL, et al. Pediatric pulmonary hypertension guidelines from the American Heart Association and American Thoracic Society. Circulation 2015; 132: 2037-99.

6. Balaguru D, Dilawar M. Pulmonary atresia with ventricular septal defect: systematic review. Heart Views 2007; 8: 52-6.

7. Charan J, Biswas T. How to calculate sample size for different study designs in medical research? Indian J Psychol Med 2013; 35: 121-6.

8. Look JE, Keane JF, Perry SB. Diagnostic and interventional catheterization in congenital heart disease. $2^{\text {nd }}$ ed. Kluwer Academic 2000; 3: 32-72.

9. Piehler JM, Danielson GK, McGoon DC, Wallace RB, Fulton RE, Mair DD. Management of pulmonary atresia with ventricular septal defect and hypoplastic pulmonary arteries by right ventricular outflow construction. J Thorac Cardiovasc Surg 1980; 80: 552-67.

10. Sultan M, Ullah M, Sadiq N, Akhtar K, Ahmed S. Diagnostic cardiac catheterization in patients of tetralogy of Fallot - prograde vs. retrograde approach. Pak Heart J 2013; 46: 39-43.

11. Saxena A, Mehta A, Sharma M, et al. Birth prevalence of congenital heart disease: a cross-sectional observational study from North India. Ann Pediatr Cardiol 2016; 9: 205-9.

12. Bassili A, Mokhtar SA, Dabous NI, Zaher SR, Mokhtar MM, Zaki A. Risk factors for congenital heart diseases in Alexandria, Egypt. Eur J Epidemiol 2000; 16: 805-14.

13. Kumar R. Congenital heart disease profile: four perspectives. Ann Pediatr Cardiol 2016; 9: 2013-4.

14. Groh GK, Levy PT, Holland MR, et al. Doppler echocardiography inaccurately estimates right ventricular pressure in children with elevated right heart pressure. J Am Soc Echocardiogr 2014; 27: 342-3.

15. Taleb M, Khuder S, Tinkle J, Khouri SJ. The diagnostic accuracy of Doppler echocardiography in assessment of pulmonary artery systolic pressure: a meta-analysis. Echocardiography 2013; 30: 258-65. 
16. Sohrabi B, Kazemi B, Mehryar A, et al. Correlation between pulmonary artery pressure measured by echocardiography and right heart catheterization in patients with rheumatic mitral valve stenosis (a prospective study). Echocardiography 2016; 33: 7-13.

17. Myers PO, Tissot C, Beghetti M. Assessment of operability of patients with pulmonary hypertention. Circ J 2014; 78: 4-11.

18. Beghetti M, Galiè N, Bonnet D. Can "inoperable" congenital heart defects become operable in patients with pulmonary pulmonary arterial hypertension? Dream or reality? Congenit Heart Dis 2012; 7: 3-11.

19. Fogel MA, Donofrio MT, Ramaciotti C, Hubbard AM, Weinberg PM. Magnetic resonance and echocardiographic imaging of pulmonary artery size throughout stages of Fontan reconstruction. Circulation 1994; 90 : 2927-36.

20. Hayabuchi Y, Inoue M, Watanabe N, Sakata M, Nabo MM, Kitagawa T. Assessment of systemic - pulmonary collateral arteries in children with cyanotic congenital heart disease using multidetector - row computed tomography: comparison with conventional angiography. Int J Cardiol 2010; 138: 266-71.

21. Zakaria RH, Barsoum NR, Asaad RE, El-Basmy AA, Azab AO. Tetralogy of Fallot: imaging of common and uncommon associations by multidetector CT. Egypt J Radiol Nuclear Med 2011; 42: 289-95.

22. Moustafa GA, Kolokythas A, Charitakis K, Avgerinos DV. Diagnostic cardiac catheterization in the pediatric population. Curr Cardiol Rev 2016; 12: 155-62.

23. Kumar P, Joshi VS, Madhu PV. Diagnostic pediatric cardiac catheterization: experience of a tertiary care pediatric cardiac centre. Med J Armed Forces India 2014; 70: 10-6.

24. Brotmacher L, Campbell M. ventricular septal defect with pulmonary stenosis. Br Heart J 1958; 20: 379-88.

25. Yousef M, Ahmed B, Tamboul J, et al. The role of cardia catheterization in the diagnosis and assessment of heart disease in Khartoum. Kasmera 2016; 44: 1-3. 